\title{
Sensitivity of the KM3NeT/ORCA detector to the neutrino mass ordering and beyond
}

\section{Mathieu Perrin-Terrin ${ }^{a, *}$ on behalf of the KM3NeT Collaboration}

(a complete list of authors can be found at the end of the proceedings)

${ }^{a}$ Aix Marseille Univ, CNRS/IN2P3, CPPM, Marseille, France

E-mail: mathieu.perrin-terrin@cppm.in2p3.fr

The KM3NeT collaboration is currently building a new generation of large-volume waterCherenkov neutrino telescopes in the Mediterranean sea. Two detectors, ARCA and ORCA, are under construction. They feature different neutrino energy thresholds: TeV range for ARCA and $\mathrm{GeV}$ range for ORCA. The main research goal of ORCA is the measurement of the neutrino mass ordering and atmospheric neutrino oscillation parameters, while the detector is also sensitive to a wide variety of other physics topics, including non-standard interactions, sterile neutrinos and Earth tomography, as well as low-energy neutrino astronomy. This contribution will present an overview of the updated ORCA sensitivity projection to its main science objectives, including - but not limited to - the measurement of the neutrino mass ordering and oscillation parameters Future perspectives for ORCA to serve as far detector for a long baseline neutrino experiment with a neutrino beam from the U70 accelerator complex at Protvino in Russia will also be discussed.

$37^{\text {th }}$ International Cosmic Ray Conference (ICRC 2021)

July 12 th - 23rd, 2021

Online - Berlin, Germany

\footnotetext{
${ }^{*}$ Presenter
} 


\section{Introduction}

The KM3NeT collaboration is building the next-generation large-volume neutrino telescopes at the bottom of the Mediterranean sea at $\sim 3 \mathrm{~km}$ depth [1]. The detectors are constructed as 3-dimensional grids of Digital Optical Modules (DOMs), each DOM housing 31 photomultiplier tubes (PMTs) for the detection of Cherenkov light emitted by charged particles emerging from from neutrino interactions in sea water. The DOMs are attached to Detection Units (DUs), which are vertical strings anchored to the seabed. Each DU holds 18 DOMs. The spacing between the DOMs on a DU and the DUs themselves is configurable. The KM3NeT/ARCA detector is being built off-coast Italy and will instrument $\sim 1 \mathrm{~km}^{3}$ of sea water with two blocks of $115 \mathrm{DU}$, with the main physics goal of detection of TeV-scale neutrinos from cosmic sources. The KM3NeT/ORCA detector is being built off-coast France and will instrument $\sim 5.5 \times 10^{-3} \mathrm{~km}^{3}$ of sea water with 115 DU and with smaller spacing between the DOMs compared to KM3NeT/ARCA. The main physics goal of ORCA is to determine the neutrino mass ordering (NMO). This contribution focuses on the KM3NeT/ORCA detector, its sensitivity to the NMO and other oscillation phenomena, and its potential use as a far detector for a long baseline neutrino experiment of a new kind with a tagged neutrino beam from U70 in Russia. The results reported here refer to the full detector. Note that the modular design of the detector allows to collect and analyse data already during the construction.

\section{Sensitivity to the oscillation parameters using atmospheric neutrinos}

The method to estimate the sensitivity to neutrino oscillation parameters is described in details in [2]. These parameters are measured by using the modifiations they induce on the distributions of the neutrino events in the plane energy - cosine zenith angle. The efficiency to detect, reconstruct and select the neutrino to be analysed is graphically reported in Figure 1 as the effective volume of the detector.

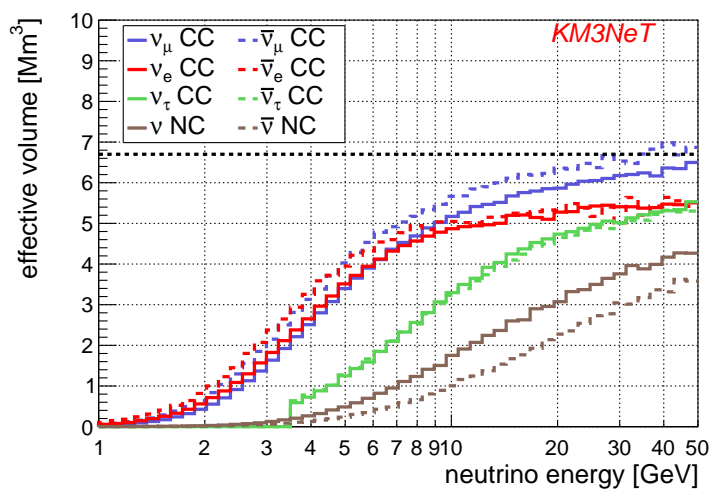

Figure 1: Effective detector volume as a function of true neutrino energy $E_{v}$ for different neutrino flavours and interactions. The dashed black line indicates the instrumented volume of the detector.

The sample of neutrino events are split in three populations using a classifier trained to separate track-like events from shower-like ones. The three classes used are named track, shower and intermediate and contains respectively a majority of track-like events, shower-like events and an admixture of the two. The performance of the classifier is illustrated in Figure 2. 

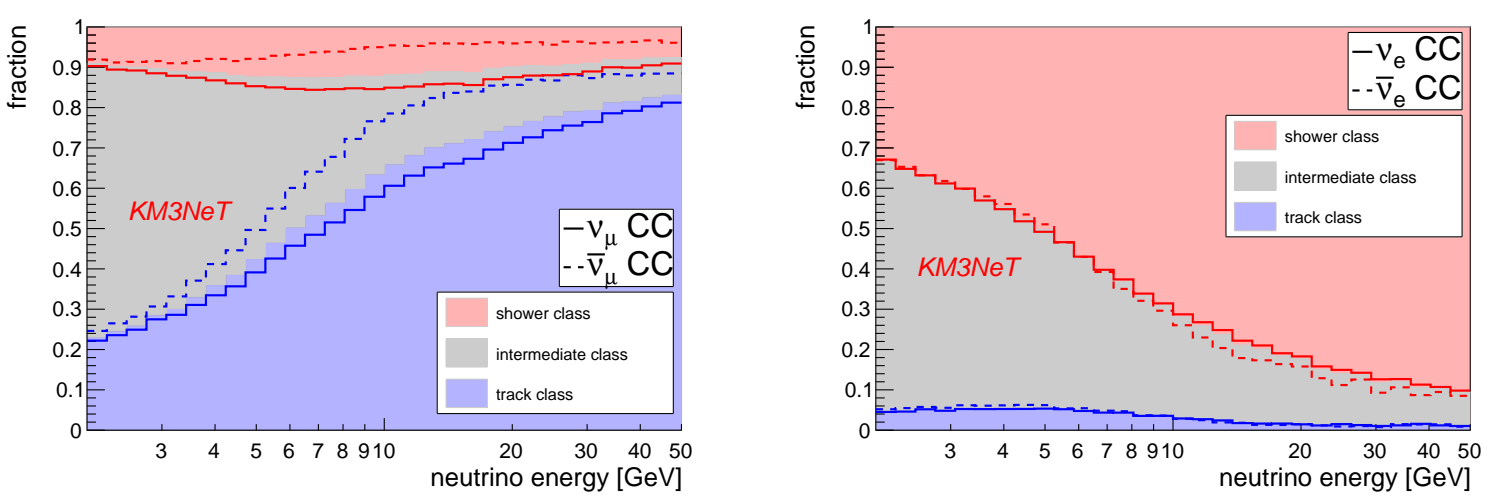

Figure 2: Fractions of preselected neutrino events of different types that are classified in the track class, the intermediate class, and the shower class, as a function of true neutrino energy. Coloured areas correspond to the composition of the atmospheric neutrino flux. Solid and dashed lines show individual fractions for neutrinos and anti-neutrinos, respectively.

The resolution on the neutrino energy is $\Delta E / E \approx 25 \%$ for $\stackrel{\mathrm{c}_{e}}{v_{e}} \mathrm{CC}$ events with $E_{v}=10 \mathrm{GeV}$, and it is dominated by the intrinsic light yield fluctuations in the hadronic shower [3]. For ${ }^{c} \vec{v}_{\mu} \mathrm{CC}$, the resolution on the neutrino energy levels off at $\Delta E / E \approx 35 \%$ as the reconstructed muon track tends not to be fully contained inside the instrumented volume.

The median neutrino direction resolution is dominated by the intrinsic $v$-lepton scattering kinematics [3] and is $9.3^{\circ} / 7.0^{\circ} / 8.3^{\circ} / 6.5^{\circ}$ for $v_{\mathrm{e}} / \bar{v}_{e} / v_{\mu} / \bar{v}_{\mu} \mathrm{CC}$ events, respectively.

Based on the detector response, on the neutrino flux and on the oscillation probabilities a model of the experiment is built to estimate the sensitivity to various observables. This model is used to generate Asimov data-sets representing the average data-sets expected under any given sets of oscillation parameters. The compatibility between each of these datasets and any other hypothetical oscillation parameter value is assessed by fitting the data-set with the experiment model corresponding to this hypothesis. During the fitting procedure several systematic uncertainties related to the neutrino flux, the detector response and the oscillation parameters are considered by allowing some of the model parameters to vary. The full description of these parameters is available in [2].

\subsection{Neutrino mass ordering}

The sensitivity to the NMO after three years of data taking is reported as a function of $\theta_{23}$ for both NMO in Figure 3. Assuming the current best estimates for $\theta_{23}$, the NMO sensitivity is $4.4 \sigma$ if the true NMO is NO and $2.3 \sigma$ if it is IO.

Figure 3 shows also the sensitivity for both NMO as a function of data taking time. The NMO can be determined at $3 \sigma$ level after 1.3 years if the true NMO is $\mathrm{NO}$, and after 5.0 years if it is IO.

\subsection{Oscillation parameters}

The atmospheric neutrino collected with KM3NeT-ORCA will also allow to measure $\Delta m_{32}^{2}$ and $\theta_{23}$. The $90 \%$ confidence level contours on these parameters for both NMO are shown in Figure 4. The $90 \%$ confidence level interval on $\Delta m_{32}^{2}$ and $\theta_{23}$ are $85 \cdot 10^{-6} \mathrm{eV}^{2}$ and $\left(\begin{array}{l}+1.9 \\ -1.1\end{array}\right)^{\circ}$ for NO and, $75 \cdot 10^{-6} \mathrm{eV}^{2}$ and $\left({ }_{-7.0}^{+2.0}\right)^{\circ}$ for IO. 

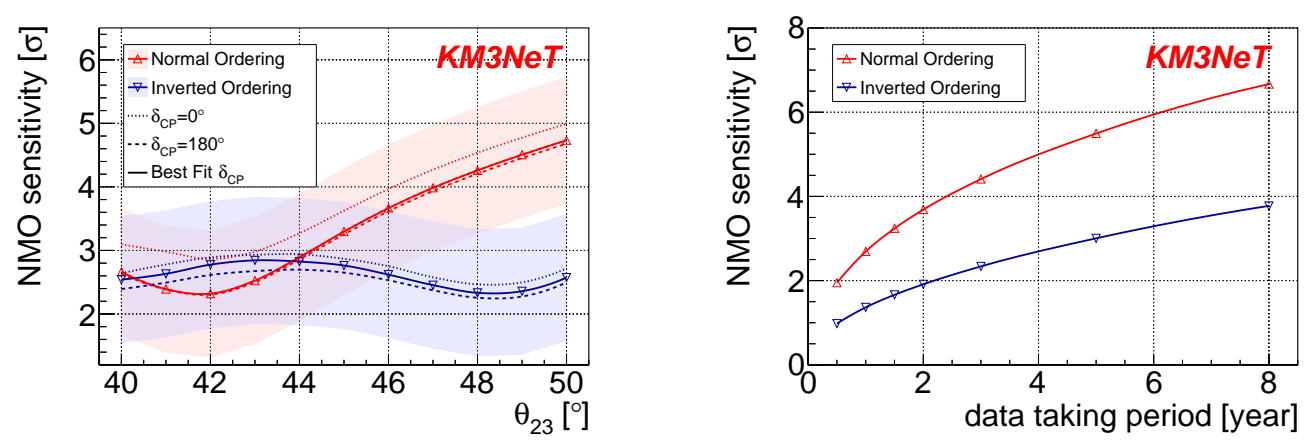

Figure 3: (left) Sensitivity to NMO after three years of data taking, as a function of the true $\theta_{23}$ value, for both normal (red upward pointing triangles) and inverted ordering (blue downward pointing triangles) under three assumptions for the $\delta_{\mathrm{CP}}$ value: the world best fit point for NO, IO (plain line), $0^{\circ}$ (dotted line) or $180^{\circ}$ (dashed line). The coloured shaded areas represent the sensitivity that $68 \%$ of the experiment realisation would yield, according to the Asimov approach [7]. (right) Sensitivity to NMO as a function of data taking time for both normal (red upward pointing triangles) and inverted ordering (blue downward pointing triangles) and assuming the oscillation parameters reported in [4].
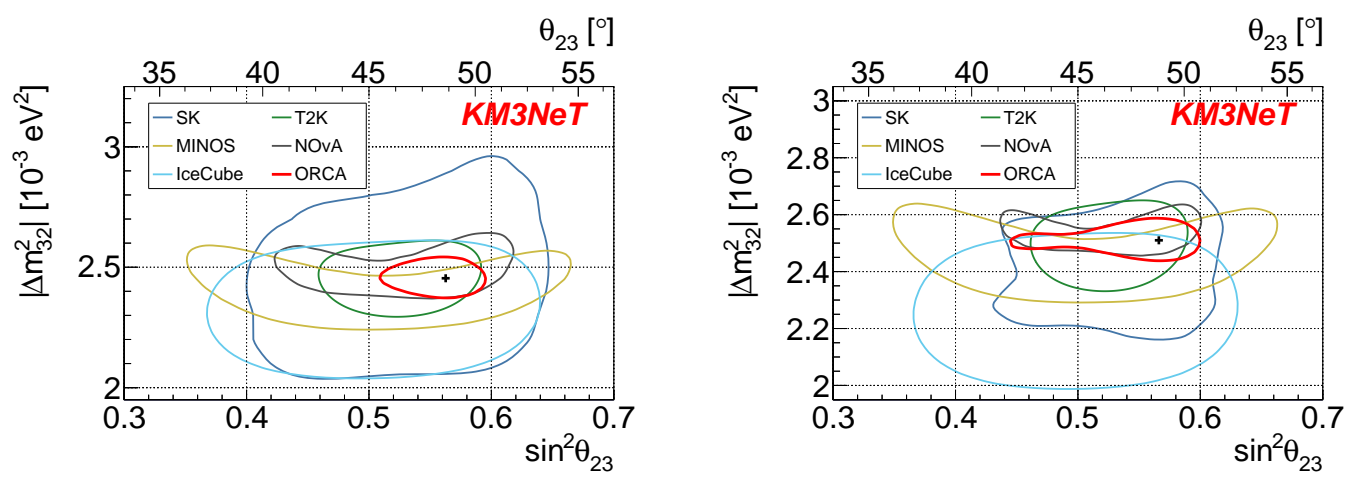

Figure 4: Expected measurement precision of $\Delta m_{32}^{2}$ and $\theta_{23}$ for both NO (left) and IO (right) after 3 years of data taking at $90 \%$ confidence level (red) overlaid with results from other experiments [8-12] and the oscillation parameters reported in [4] (black cross).

The same analysis allows to calculate the significance to determine the octant of $\theta_{23}$. The results are shown in Figure 5, which illustrates the needed data taking time to reach a 1,2 and $3 \sigma$ octant significance as a function of the true value of $\theta_{23}$. Dashed lines ignore the NMO, while for solid lines the NMO is assumed to be known.

\section{$2.3 v_{\tau}$ appearance}

Finally the unitarity of the PMNS matrix will be tested using the ${ }^{\mathrm{c}} \vec{v}_{\mu} \rightarrow{ }^{\mathrm{c}} \vec{v}_{\tau}$ channel. About $3000{ }^{c} \vec{v}_{\tau}$ per year will be collected with the full detector. The appearance of ${ }^{c} \vec{v}_{\tau}$ is determined by measuring the normalisation factor of the ${ }^{c_{v}}{ }_{\tau}$ contribution. For this study, NO is assumed. In Figure 6, the sensitivity is presented as a function of operation time. KM3NeT/ORCA will already be able to confirm the exclusion of non-appearance with high statistical significance with few months of data-taking. The normalisation can be constrained to $\pm 30 \%$ at $3 \sigma$-level and to $\pm 10 \%$ at $1 \sigma$-level after one year of data taking. 

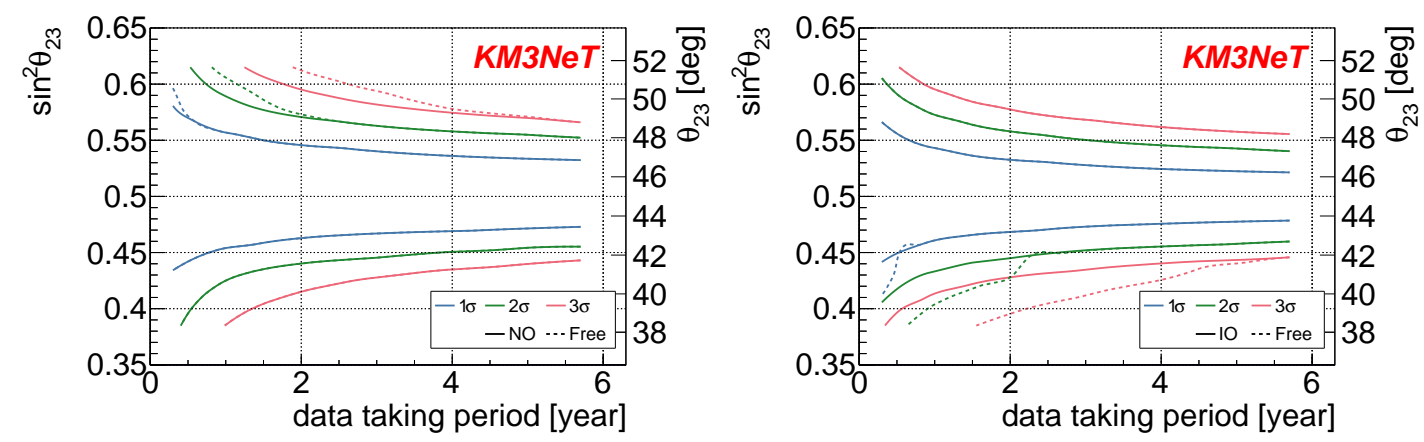

Figure 5: Expected sensitivity to determine the $\theta_{23}$ octant at 1 (blue), 2 (green) or $3 \sigma$ (red) as a function of data taking time for both NO (left) and IO (right) assuming the true NMO is known (solid line) or unknown (dashed line). The dashed lines differ from the plain ones when the fit converges to the wrong NMO.

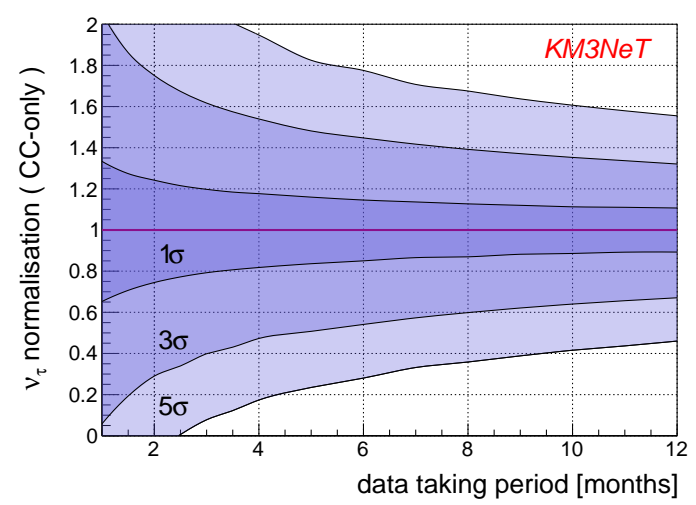

Figure 6: Sensitivity to ${ }^{c} \vec{v}_{\tau}$ appearance as a function of data taking period.

\section{A new kind of long baseline neutrino experiment}

The precise determination of the $\mathrm{CP}$ violating phase, $\delta_{\mathrm{CP}}$, is one of the keystones of neutrino physics in the coming decade. KM3NeT-ORCA could be used to make this measurement as a far detector in a long baseline neutrino experiment (LBLNE) with a neutrino beam produced in U70 at Protvino in Russia. A first version of this project, called P2O is described in [13].

Given the large mass of KM3NeT-ORCA, compared to other future LBLNE [14, 15], a modest beam intensity of $O(100) \mathrm{kW}$ is sufficient to collect a large sample of neutrino: $O\left(10^{3}\right)$ per year. At these intensities, it appears feasible to instrument the beam line with trackers. These trackers would allow to reconstruct kinematically the properties of the neutrinos produced by $\pi^{ \pm} \rightarrow \mu^{ \pm} v$ decays. Energy resolutions better than $1 \%$ are accessible with this method. In addition, each and all neutrinos from $\pi^{ \pm} \rightarrow \mu^{ \pm} v$ are reconstructed individually which removes the systematic uncertainties on the beam composition. Finally, provided that the far detector is synchronized with the trackers, the property of the neutrino kinematically reconstructed can be assigned to the interacting neutrino. The far detector is thus left with the unique task of identifying the flavor of the neutrino after propagation. In addition, as the chirality is determined by the trackers, both neutrino and anti-neutrino can be collected together which helps to resolve the $\theta_{23}-\delta_{\mathrm{CP}}$ degeneracy. More details on this method can be found in [16, 17]. 
The precision on $\delta_{\mathrm{CP}}$ is represented as function of $\delta_{\mathrm{CP}}$ in Figure 7 (left) together with the expectation for DUNE assuming a $450 \mathrm{~kW}$ beam. The precision is stable over the whole span of $\delta_{\mathrm{CP}}$ values and ranges between $6^{\circ}$ and $8^{\circ}$. These two extreme values are obtained for 0 and $90^{\circ}$. The precision at these two values is shown as a function of the exposure in Figure 7 (right). Overall, P2O with a tagged beam appears a valuable and complementary approach to next generation of LBLNE.
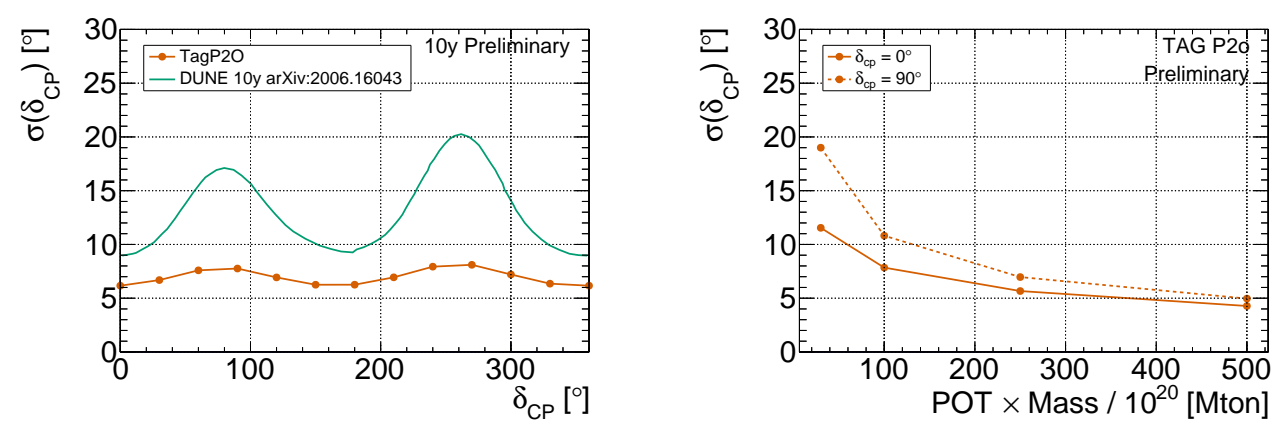

Figure 7: (left) Precision to measure $\delta_{\mathrm{CP}}$ as function of $\delta_{\mathrm{CP}}$ and (right) as function of exposure.

\section{References}

[1] S. Adrian-Martinez et al., J. Phys. G43(8), 084001 (2016).

[2] S. Aiello et al., arXiv:2103.09885v1

[3] S. Adrián-Martínez, et al., JHEP 05, 008 (2017).

[4] I. Esteban, et al., DOI 10.1007/JHEP01(2019)106

[5] L. Wolfenstein, Phys. Rev. D 17, 2369 (1978). DOI 10.1103/PhysRevD.17.2369

[6] S.P. Mikheyev, A.Y. Smirnov, Sov. J. Nucl. Phys. 42, 913 (1985)

[7] G. Cowan et al., Eur. Phys. J. C 71, 1554 (2011). [Erratum: Eur.Phys.J.C 73, 2501 (2013)]

[8] M. G. Aartsen et al., Phys. Rev. Lett. 120(7), 071801 (2018).

[9] K. Abe et al., Phys. Rev. D 97(7), 072001 (2018).

[10] K. Abe et al., Nature 580(7803), 339 (2020).

[11] A. Aurisano. Recent Results from MINOS and MINOS+ (2018).

[12] M. A. Acero et al., Phys. Rev. Lett. 123(15), 151803 (2019).

[13] A.V. Akindinov et al., Eur. Phys. J. C79(9), 758 (2019).

[14] B. Abi et al., arXiv:1807.10334

[15] K. Abe et al., arXiv:1805.04163

[16] M. Perrin-Terrin, NeuTel 2021, https://doi.org/10.5281/zenodo.4912631

[17] M. Perrin-Terrin, VLVnT 2021, https://indico.ific.uv.es/event/3965/contributions/14787/ 


\section{Full Author List: KM3NeT Collaboration}

M. Ageron ${ }^{1}$, S. Aiello ${ }^{2}$, A. Albert ${ }^{3,55}$, M. Alshamsi ${ }^{4}$, S. Alves Garre ${ }^{5}$,Z. Aly ${ }^{1}$, A. Ambrosone ${ }^{6,7}$, F. Ameli ${ }^{8}$, M. Andre ${ }^{9}$, G. Androulakis ${ }^{10}$, M. Anghinolfi ${ }^{11}$, M. Anguita ${ }^{12}$, G. Anton ${ }^{13}$, M. Ardid ${ }^{14}$, S. Ardid ${ }^{14}$, W. Assal ${ }^{1}$, J. Aublin ${ }^{4}$, C. Bagatelas ${ }^{10}$, B. Baret ${ }^{4}$, S. Basegmez du Pree ${ }^{15}$, M. Bendahman ${ }^{4,16}$, F. Benfenati ${ }^{17,18}$, E. Berbee ${ }^{15}$, A. M. van den Berg ${ }^{19}$, V. Bertin ${ }^{1}$, S. Beurthey ${ }^{1}$, V. van Beveren ${ }^{15}$, S. Biagi ${ }^{20}$, M. Billault ${ }^{1}$, M. Bissinger ${ }^{13}$, M. Boettcher ${ }^{21}$, M. Bou Cabo ${ }^{22}$, J. Boumaaza ${ }^{16}$, M. Bouta ${ }^{23}$, C. Boutonnet ${ }^{4}$, G. Bouvet ${ }^{24}$, M. Bouwhuis ${ }^{15}$, C. Bozza ${ }^{25}$, H.Brânzaş ${ }^{26}$, R. Bruijn ${ }^{15,27}$, J. Brunner ${ }^{1}$, R. Bruno ${ }^{2}$, E. Buis ${ }^{28}$, R. Buompane ${ }^{6,29}$, J. Busto ${ }^{1}$, B. Caiffi ${ }^{11}$, L. Caillat ${ }^{1}$, D. Calvo ${ }^{5}$, S. Campion ${ }^{30,8}$, A. Capone ${ }^{30,8}$, H. Carduner $^{24}$, V. Carretero ${ }^{5}$, P. Castaldi ${ }^{17,31}$, S. Celli ${ }^{30,8}$, R. Cereseto ${ }^{11}$, M. Chabab ${ }^{32}$, C. Champion ${ }^{4}$, N. $\mathrm{Chau}^{4}$, A. Chen ${ }^{33}$, S. Cherubini ${ }^{20,34}$, V. Chiarella ${ }^{35}$, T. Chiarusi ${ }^{17}$, M. Circella ${ }^{36}$, R. Cocimano ${ }^{20}$, J. A. B. Coelho ${ }^{4}$, A. Coleiro ${ }^{4}$, M. Colomer Molla ${ }^{4,5}$, S. Colonges ${ }^{4}$, R. Coniglione ${ }^{20}$, A. Cosquer ${ }^{1}$, P. Coyle ${ }^{1}$, M. Cresta ${ }^{11}$, A. Creusot ${ }^{4}$, A. $\mathrm{Cruz}^{37}$, G. Cuttone ${ }^{20}$, A. D’Amico ${ }^{15}$, R. Dallier ${ }^{24}$, B. De Martino ${ }^{1}$, M. De Palma ${ }^{36,38}$, I. Di Palma ${ }^{30,8}$, A. F. Díaz ${ }^{12}$, D. Diego$\operatorname{Tortosa}^{14}$, C. Distefano ${ }^{20}$, A. Domi ${ }^{15,27}$, C. Donzaud ${ }^{4}$, D. Dornic ${ }^{1}$, M. Dörr ${ }^{39}$, D. Drouhin ${ }^{3,55}$, T. Eberl ${ }^{13}$, A. Eddyamoui ${ }^{16}$, T. van Eeden ${ }^{15}$, D. van Eijk ${ }^{15}$, I. El Bojaddaini ${ }^{23}$, H. Eljarrari ${ }^{16}$, D. Elsaesser ${ }^{39}$, A. Enzenhöfer ${ }^{1}$, V. Espinosa ${ }^{14}$, P. Fermani ${ }^{30,8}$, G. Ferrara ${ }^{20,34}$, M. D. Filipović ${ }^{40}$, F. Filippini ${ }^{17,18}$, J. Fransen ${ }^{15}$, L. A. Fusco ${ }^{1}$, D. Gajanana ${ }^{15}$, T. Gal ${ }^{13}$, J. García Méndez ${ }^{14}$, A. Garcia Soto ${ }^{5}$, E. Garçon ${ }^{1}$, F. Garufi ${ }^{6,7}$, C. Gatius ${ }^{15}$, N. Geißelbrecht ${ }^{13}$, L. Gialanella ${ }^{6,29}$, E. Giorgio ${ }^{20}$, S. R. Gozzini ${ }^{5}$, R. Gracia ${ }^{15}$, K. Graf ${ }^{13}$, G. Grella ${ }^{41}$, D. Guderian ${ }^{56}$, C. Guidi ${ }^{11,42}$, B. Guillon ${ }^{43}$, M. Gutiérrez ${ }^{44}$, J. Haefner ${ }^{13}$, S. Hallmann ${ }^{13}$, H. Hamdaoui ${ }^{16}$, H. van Haren ${ }^{45}$, A. Heijboer ${ }^{15}$, A. Hekalo ${ }^{39}$, L. Hennig ${ }^{13}$, S. Henry ${ }^{1}$, J. J. Hernández-Rey ${ }^{5}$, J. Hofestädt ${ }^{13}$, F. Huang ${ }^{1}$, W. Idrissi Ibnsalih ${ }^{6,29}$, A. Ilioni ${ }^{4}$, G. Illuminati ${ }^{17,18,4}$, C. W. James ${ }^{37}$, D. Janezashvili ${ }^{46}$, P. Jansweijer ${ }^{15}$, M. de Jong ${ }^{15,47}$, P. de Jong ${ }^{15,27}$, B. J. Jung ${ }^{15}$, M. Kadler ${ }^{39}$, P. Kalaczyński ${ }^{48}$, O. Kalekin ${ }^{13}$, U. F. Katz ${ }^{13}$, F. Kayzel ${ }^{15}$, P. Keller ${ }^{1}$, N. R. Khan Chowdhury ${ }^{5}$, G. Kistauri ${ }^{46}$, F. van der Knaap ${ }^{28}$, P. Kooijman ${ }^{27,57}$, A. Kouchner ${ }^{4,49}$, M. Kreter ${ }^{21}$, V. Kulikovskiy ${ }^{11}$, M. Labalme ${ }^{43}$, P. Lagier ${ }^{1}$, R. Lahmann ${ }^{13}$, P. Lamare ${ }^{1}$, M. Lamoureux $^{14}$, G. Larosa ${ }^{20}$, C. Lastoria ${ }^{1}$, J. Laurence ${ }^{1}$, A. Lazo $^{5}$, R. Le Breton ${ }^{4}$, E. Le Guirriec ${ }^{1}$, S. Le Stum ${ }^{1}$, G. Lehaut ${ }^{43}$, O. Leonardi ${ }^{20}$, F. Leone ${ }^{20,34}$, E. Leonora ${ }^{2}$, C. Lerouvillois ${ }^{1}$, J. Lesrel ${ }^{4}$, N. Lessing ${ }^{13}$, G. Levi ${ }^{17,18}$, M. Lincetto ${ }^{1}$, M. Lindsey Clark ${ }^{4}$, T. Lipreau ${ }^{24}$, C. LLorens Alvarez ${ }^{14}$, A. Lonardo ${ }^{8}$, F. Longhitano ${ }^{2}$, D. Lopez-Coto ${ }^{44}$, N. Lumb ${ }^{1}$, L. Maderer ${ }^{4}$, J. Majumdar ${ }^{15}$, J. Mańczak ${ }^{5}$, A. Margiotta ${ }^{17,18}$, A. Marinelli ${ }^{6}$, A. Marini ${ }^{1}$, C. Markou $^{10}$, L. Martin ${ }^{24}$, J. A. Martínez-Mora ${ }^{14}$, A. Martini ${ }^{35}$, F. Marzaioli ${ }^{6,29}$,

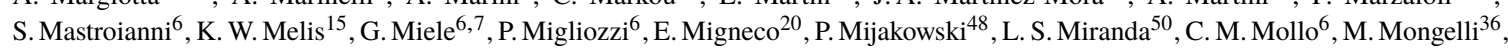
A. Moussa ${ }^{23}$, R. Muller ${ }^{15}$, P. Musico ${ }^{11}$, M. Musumeci ${ }^{20}$, L. Nauta ${ }^{15}$, S. Navas ${ }^{44}$, C. A. Nicolau ${ }^{8}$, B. Nkosi ${ }^{33}$, B. Ó Fearraigh ${ }^{15,27}$, M. O'Sullivan ${ }^{37}$, A. Orlando ${ }^{20}$, G. Ottonello ${ }^{11}$, S. Ottonello ${ }^{11}$, J. Palacios González ${ }^{5}$, G. Papalashviliit ${ }^{46}$, R. Papaleo ${ }^{20}$, C. Pastore ${ }^{36}$, A. M. Păun ${ }^{26}$, G. E. Păvălaş ${ }^{26}$, G. Pellegrini ${ }^{17}$, C. Pellegrino ${ }^{18,58}$, M. Perrin-Terrin ${ }^{1}$, V. Pestel ${ }^{15}$, P. Piattelli ${ }^{20}$, C. Pieterse ${ }^{5}$, O. Pisanti ${ }^{6,7}$, C. Poirè ${ }^{14}$, V. Popa $^{26}$, T. Pradier $^{3}$, F. Pratolongo ${ }^{11}$, I. Probst ${ }^{13}$, G. Pühlhofer ${ }^{51}$, S. Pulvirenti ${ }^{20}$, G. Quéméner ${ }^{43}$, N. Randazzo ${ }^{2}$, A. Rapicavoli ${ }^{34}$, S. Razzaque $^{50}$, D. Real ${ }^{5}$, S. Reck ${ }^{13}$, G. Riccobene ${ }^{20}$, L. Rigalleau ${ }^{24}$, A. Romanov ${ }^{11,42}$, A. Rovelli ${ }^{20}$, J. Royon ${ }^{1}$, F. Salesa Greus ${ }^{5}$, D. F. E. Samtleben ${ }^{15,47}$, A. Sánchez Losa ${ }^{36,5}$, M. Sanguineti ${ }^{11,42}$, A. Santangelo ${ }^{51}$, D. Santonocito ${ }^{20}$, P. Sapienza ${ }^{20}$, J. Schmelling ${ }^{15}$, J. Schnabel ${ }^{13}$, M. F. Schneider ${ }^{13}$, J. Schumann ${ }^{13}$, H. M. Schutte ${ }^{21}$, J. Seneca ${ }^{15}$, I. Sgura ${ }^{36}$, R. Shanidze ${ }^{46}$, A. Sharma ${ }^{52}$, A. Sinopoulou ${ }^{10}$, B. Spisso ${ }^{41,6}$, M. Spurio ${ }^{17,18}$, D. Stavropoulos ${ }^{10}$, J. Steijger ${ }^{15}$, S. M. Stellacci ${ }^{41,6}$, M. Taiuti ${ }^{11,42}$, F. Tatone ${ }^{36}$, Y. Tayalati ${ }^{16}$, E. Tenllado ${ }^{44}$, D. Tézier ${ }^{1}$, T. Thakore ${ }^{5}$, S. Theraube ${ }^{1}$, H. Thiersen $^{21}$, P. Timmer $^{15}$, S. Tingay ${ }^{37}$, S. Tsagkli $^{10}$, V. Tsourapis ${ }^{10}$, E. Tzamariudaki ${ }^{10}$, D. Tzanetatos ${ }^{10}$, C. Valieri ${ }^{17}$, V. Van Elewyck ${ }^{4,49}$, G. Vasileiadis ${ }^{53}$, F. Versari ${ }^{17,18}$, S. Viola $^{20}$, D. Vivolo ${ }^{6,29}$, G. de Wasseige ${ }^{4}$, J. Wilms ${ }^{54}$, R. Wojaczyński ${ }^{48}$, E. de Wolf ${ }^{15,27}$, T. Yousfi ${ }^{23}$, S. Zavatarelli ${ }^{11}$, A. Zegarelli ${ }^{30,8}$, D. Zito ${ }^{20}$, J. D. Zornoza ${ }^{5}$, J. Zúñiga ${ }^{5}$, N. Zywucka ${ }^{21}$.

${ }^{1}$ Aix Marseille Univ, CNRS/IN2P3, CPPM, Marseille, France.

${ }^{2}$ INFN, Sezione di Catania, Via Santa Sofia 64, Catania, 95123 Italy.

${ }^{3}$ Université de Strasbourg, CNRS, IPHC UMR 7178, F-67000 Strasbourg, France.

${ }^{4}$ Université de Paris, CNRS, Astroparticule et Cosmologie, F-75013 Paris, France.

${ }^{5}$ IFIC - Instituto de Física Corpuscular (CSIC - Universitat de València), c/Catedrático José Beltrán, 2, 46980 Paterna, Valencia, Spain. ${ }^{6}$ INFN, Sezione di Napoli, Complesso Universitario di Monte S. Angelo, Via Cintia ed. G, Napoli, 80126 Italy.

${ }^{7}$ Università di Napoli “Federico II”, Dip. Scienze Fisiche "E. Pancini”, Complesso Universitario di Monte S. Angelo, Via Cintia ed. G, Napoli, 80126 Italy.

${ }^{8}$ INFN, Sezione di Roma, Piazzale Aldo Moro 2, Roma, 00185 Italy.

${ }^{9}$ Universitat Politècnica de Catalunya, Laboratori d'Aplicacions Bioacústiques, Centre Tecnològic de Vilanova i la Geltrú, Avda. Rambla Exposició, s/n, Vilanova i la Geltrú, 08800 Spain.

${ }^{10}$ NCSR Demokritos, Institute of Nuclear and Particle Physics, Ag. Paraskevi Attikis, Athens, 15310 Greece.

${ }^{11}$ INFN, Sezione di Genova, Via Dodecaneso 33, Genova, 16146 Italy.

${ }^{12}$ University of Granada, Dept. of Computer Architecture and Technology/CITIC, 18071 Granada, Spain.

${ }^{13}$ Friedrich-Alexander-Universität Erlangen-Nürnberg, Erlangen Centre for Astroparticle Physics, Erwin-Rommel-Straße 1, 91058 Erlangen, Germany.

${ }^{14}$ Universitat Politècnica de València, Instituto de Investigación para la Gestión Integrada de las Zonas Costeras, C/ Paranimf, 1, Gandia, 46730 Spain.

${ }^{15}$ Nikhef, National Institute for Subatomic Physics, PO Box 41882, Amsterdam, 1009 DB Netherlands.

${ }^{16}$ University Mohammed V in Rabat, Faculty of Sciences, 4 av. Ibn Battouta, B.P. 1014, R.P. 10000 Rabat, Morocco.

${ }^{17}$ INFN, Sezione di Bologna, v.le C. Berti-Pichat, 6/2, Bologna, 40127 Italy.

\footnotetext{
${ }^{1}$ also at Dipartimento di Fisica, INFN Sezione di Padova and Università di Padova, I-35131, Padova, Italy
} 
${ }^{18}$ Università di Bologna, Dipartimento di Fisica e Astronomia, v.le C. Berti-Pichat, 6/2, Bologna, 40127 Italy.

${ }^{19}$ KVI-CART University of Groningen, Groningen, the Netherlands.

${ }^{20}$ INFN, Laboratori Nazionali del Sud, Via S. Sofia 62, Catania, 95123 Italy.

${ }^{21}$ North-West University, Centre for Space Research, Private Bag X6001, Potchefstroom, 2520 South Africa.

${ }^{22}$ Instituto Español de Oceanografía, Unidad Mixta IEO-UPV, C/ Paranimf, 1, Gandia, 46730 Spain.

${ }^{23}$ University Mohammed I, Faculty of Sciences, BV Mohammed VI, B.P. 717, R.P. 60000 Oujda, Morocco.

${ }^{24}$ Subatech, IMT Atlantique, IN2P3-CNRS, Université de Nantes, 4 rue Alfred Kastler - La Chantrerie, Nantes, BP 2072244307 France.

${ }^{25}$ Università di Salerno e INFN Gruppo Collegato di Salerno, Dipartimento di Matematica, Via Giovanni Paolo II 132, Fisciano, 84084 Italy.

${ }^{26}$ ISS, Atomistilor 409, Măgurele, RO-077125 Romania

${ }^{27}$ University of Amsterdam, Institute of Physics/IHEF, PO Box 94216, Amsterdam, 1090 GE Netherlands.

${ }^{28}$ TNO, Technical Sciences, PO Box 155, Delft, 2600 AD Netherlands.

${ }^{29}$ Università degli Studi della Campania "Luigi Vanvitelli", Dipartimento di Matematica e Fisica, viale Lincoln 5, Caserta, 81100 Italy.

${ }^{30}$ Università La Sapienza, Dipartimento di Fisica, Piazzale Aldo Moro 2, Roma, 00185 Italy.

${ }^{31}$ Università di Bologna, Dipartimento di Ingegneria dell'Energia Elettrica e dell'Informazione "Guglielmo Marconi", Via dell'Università 50, Cesena, 47521 Italia.

${ }^{32}$ Cadi Ayyad University, Physics Department, Faculty of Science Semlalia, Av. My Abdellah, P.O.B. 2390, Marrakech, 40000 Morocco.

${ }^{33}$ University of the Witwatersrand, School of Physics, Private Bag 3, Johannesburg, Wits 2050 South Africa.

${ }^{34}$ Università di Catania, Dipartimento di Fisica e Astronomia "Ettore Majorana", Via Santa Sofia 64, Catania, 95123 Italy.

${ }^{35}$ INFN, LNF, Via Enrico Fermi, 40, Frascati, 00044 Italy.

${ }^{36}$ INFN, Sezione di Bari, via Orabona, 4, Bari, 70125 Italy.

${ }^{37}$ International Centre for Radio Astronomy Research, Curtin University, Bentley, WA 6102, Australia.

${ }^{38}$ University of Bari, Via Amendola 173, Bari, 70126 Italy.

${ }^{39}$ University Würzburg, Emil-Fischer-Straße 31, Würzburg, 97074 Germany.

${ }^{40}$ Western Sydney University, School of Computing, Engineering and Mathematics, Locked Bag 1797, Penrith, NSW 2751 Australia.

${ }^{41}$ Università di Salerno e INFN Gruppo Collegato di Salerno, Dipartimento di Fisica, Via Giovanni Paolo II 132, Fisciano, 84084 Italy.

${ }^{42}$ Università di Genova, Via Dodecaneso 33, Genova, 16146 Italy.

${ }^{43}$ Normandie Univ, ENSICAEN, UNICAEN, CNRS/IN2P3, LPC Caen, LPCCAEN, 6 boulevard Maréchal Juin, Caen, 14050 France.

${ }^{44}$ University of Granada, Dpto. de Física Teórica y del Cosmos \& C.A.F.P.E., 18071 Granada, Spain.

${ }^{45} \mathrm{NIOZ}$ (Royal Netherlands Institute for Sea Research), PO Box 59, Den Burg, Texel, 1790 AB, the Netherlands.

${ }^{46}$ Tbilisi State University, Department of Physics, 3, Chavchavadze Ave., Tbilisi, 0179 Georgia.

${ }^{47}$ Leiden University, Leiden Institute of Physics, PO Box 9504, Leiden, 2300 RA Netherlands.

${ }^{48}$ National Centre for Nuclear Research, 02-093 Warsaw, Poland.

${ }^{49}$ Institut Universitaire de France, 1 rue Descartes, Paris, 75005 France.

${ }^{50}$ University of Johannesburg, Department Physics, PO Box 524, Auckland Park, 2006 South Africa.

${ }^{51}$ Eberhard Karls Universität Tübingen, Institut für Astronomie und Astrophysik, Sand 1, Tübingen, 72076 Germany.

${ }^{52}$ Università di Pisa, Dipartimento di Fisica, Largo Bruno Pontecorvo 3, Pisa, 56127 Italy.

${ }^{53}$ Laboratoire Univers et Particules de Montpellier, Place Eugène Bataillon - CC 72, Montpellier Cédex 05, 34095 France.

${ }^{54}$ Friedrich-Alexander-Universität Erlangen-Nürnberg, Remeis Sternwarte, Sternwartstraße 7, 96049 Bamberg, Germany.

${ }^{55}$ Université de Haute Alsace, 68100 Mulhouse Cedex, France.

${ }^{56}$ University of Münster, Institut für Kernphysik, Wilhelm-Klemm-Str. 9, Münster, 48149 Germany.

${ }^{57}$ Utrecht University, Department of Physics and Astronomy, PO Box 80000, Utrecht, 3508 TA Netherlands.

${ }^{58}$ INFN, CNAF, v.le C. Berti-Pichat, 6/2, Bologna, 40127 Italy. 